\title{
Analysis of age of onset, pre-existing infections, and features of magnetic resonance imaging results in patients with acute myelitis
}

\author{
Q. Tang and H. Xiao \\ Neurology Department, Anhui Provincial Hospital, Hefei, Anhui, China \\ Corresponding author: Q. Tang \\ E-mail: qiqiangtang@yeah.net
}

Genet. Mol. Res. 13 (2): 4545-4551 (2014)

Received May 28, 2013

Accepted October 25, 2013

Published June 17, 2014

DOI http://dx.doi.org/10.4238/2014.June.17.6

\begin{abstract}
The clinical features and potential risk factors of acute myelitis (AM) were investigated. The medical records of patients with AM admitted to our department between January 2004 and December 2011 were collected and retrospectively analyzed. The diagnosis of AM was in line with the diagnostic criteria of the Transverse Myelitus Consortium Working Group. The age of onset, clinical, and imaging features of these patients were analyzed. A total of 64 patients satisfying the inclusion criteria were enrolled in the study, including 39 males and 25 females. The patients ranged in age from 1 to 80 years, with a mean age of 34 years. Twenty-three patients had symptoms of preexisting infections. The correlation between spinal cord lesions and spinal lesions was statistically significant $(\mathrm{P}<0.05)$. Cervical spinal cord inflammation was the most common. Prodromal infections were more commonly observed in thoracic spinal cord myelitis than in cervical spinal cord myelitis $(\mathrm{P}<0.05)$. AM appears to be more likely to occur in male minors. Lesions of the spinal column were partially implicated with the occurrence of myelitis, which suggests that such lesions might be a predisposing factor. Compared to AM of the cervical
\end{abstract}


cord, pre-existing infections appear to be of greater significance for the occurrence of myelitis of the thoracic cord.

Key words: Acute myelitis; Age of onset; Pre-existing infections; MRI

\section{INTRODUCTION}

Acute myelitis (AM), also known as acute transverse myelitis (ATM), is an acute transverse inflammatory disorder of the spinal cord that is caused by the immunological response following infections. The early use of steroid therapy could achieve partial or total recovery of spinal cord functions (Sá, 2009; Zettl et al., 2012). In spite of the establishment of comprehensive assessments and diagnostic criteria, it is still a challenge to confirm the cause of differential myelitis. There is no difference in gender, season, and family history in the occurrence of myelitis [Transverse Myelitis Consortium Working Group (TMCWG), 2002]. A Canadian study showed some difference in the incidence of AM between male and female minors (Banwell et al., 2009). However, no studies have confirmed this result in China. Moreover, since imaging examinations were employed in spinal cord assessments, some patients with AM were also found to have degeneration of inter-vertebrate disks. In a Japanese study, Matsumoto et al. (1998) found that using magnetic resonance imaging (MRI), the detection rate of diastrophic dysplasia (DD) in cervical spines of juvenile patients was positively correlated with the age. The rate of DD in the group of 10 to 19 year-old patients was lower than $5 \%$, whereas in the group of 20-29-year-old patients it was lower than $17 \%$, and in the group of 30-39-year-old patients it was lower than $25 \%$. In contrast, in the present study, the detection rates of DD in these three age groups were $85,94.1$, and $87 \%$, respectively. Although the differences in several variables, including the expertise level of imaging technicians, the diagnostic criteria used, and the MRI equipment used, between these two studies make it impossible to perform accurate statistical analysis, the large difference in these results nonetheless indicates that the detection rate of DD in cervical spines of patients with AM might be higher than that in healthy individuals. In this study, the age of onset, clinical, and imaging features of 64 hospitalized patients with AM were analyzed and the results were compared to relevant studies in order to explore the general clinical features and potential risk factors of AM.

\section{MATERIAL AND METHODS}

\section{Patients}

The medical records of patients with AM admitted from January 2004 to December 2011 in the Neurology Department of Anhui Provincial Hospital were selected. The inclusion criteria were as follows: comply with the diagnostic criteria of AM recommended by the TMCWG (2002), non-infectious signs of cerebrospinal fluid examination, and the MRI results suggest transverse disorder in the spinal cord.

The following exclusion criteria were applied: evidence of other immunological disorders, imaging examinations confirmed the existence of demyelination in the central nervous system, compression of spinal cords, and cervical trauma.

A total of 64 patients were ultimately included in the study, consisting of 39 males 
and 25 females. The patients' ages ranged from 1 to 80 years, with a mean age of 34 years, including 10 male minors (younger than 18 years of age), 30 patients between 18 and 46 years of age (14 males, 16 females), and 24 patients above 46 years of age (14 males, 10 females).

With respect to pre-existing infections and other predisposing factors, 23 patients had symptoms of pre-existing infections: 9 upper respiratory infections, 10 unknown hyperthemia infections, and 2 gastrointestinal infections. The interval between the emergence of symptoms of pre-existing infections and the occurrence of AM ranged from 7 to 30 days (mean value 10 days). In addition, 2 patients had predisposing factors: one with herpes zoster that occurred 5 days before the onset of myelitis, and the other with parotitis 10 days before the onset of myelitis (Table 1).

Table 1. Relationship between pre-existing infections and lesions of spinal cords on 64 patients with acute
myelitis.
\begin{tabular}{lccc}
\hline Lesions of spinal cord & With pre-existing infections & Without pre-existing infections & Total \\
\hline Cervical cord & 3 & 13 & 16 \\
Thoracic cord & 20 & 9 & 29 \\
\hline
\end{tabular}

\section{Imaging data}

The 64 patients were all given MRI examinations, during which signal changes were observed to detect the inflammatory disorders and lesions of spinal vertebrae (including degeneration of the spinal column and herniated disks).

Signal changes on spinal cords were observed on 18 cervical cords, 29 thoracic cords, 2 lumbar cords, 10 cases of no abnormal findings, and 5 were not available. Lesions of spinal vertebrae were observed on 18 cervical vertebrae, 3 thoracic vertebrae, 8 lumbar vertebrae, 22 had no abnormal findings, and 13 were not available (Table 2). There were 72 cases of coexistence or non-existence (both occur or neither occur) of lesions of the spinal cord and vertebrae, including 22 cases of single occurrence or at different levels, and 18 cases of unavailable data (data of either or both were unavailable). In addition, 7 patients had two lesions at most, while 19 patients had at least three lesions.

Table 2. MRI results and lesions of spinal cord on 64 patients with myelitis.
\begin{tabular}{lccccr}
\hline & Cervical & Thoracic & Lumbar & No abnormal findings & N/A \\
\hline Lesions of spinal cord & 18 & 29 & 2 & 10 & 5 \\
Lesions of spinal column & 18 & 3 & 8 & 22 & 13 \\
\hline
\end{tabular}

$\mathrm{N} / \mathrm{A}=$ not available.

\section{Statistical analysis}

Statistical analysis was conducted to evaluate the relationship between pre-existing infections and lesions of the cervical and thoracic vertebrae and cervical and thoracic myelitis, respectively. All enumeration data were analyzed using the SPSS16.0 software. All data were weighted and subjected to the chi-squared test at $\alpha=0.05$. 


\section{RESULTS}

\section{Relationship between lesions of spinal cords and vertebrae}

Twelve of the 18 patients with lesions of the cervical cord had diseases of the surrounding vertebral column, none had diseases of the spinal column, and 4 showed no abnormal findings. Five patients had diseases of the thoracic cord, 8 had no diseases of the surrounding vertebral column, and 11 showed no abnormal findings. The chi-squared test showed that lesions of spinal cords were significantly related to surrounding vertebral column infections $(\mathrm{P}<0.05)$. Although AM is generally believed to be a disease caused by the immune response occurring after infections in clinical settings, the lesions of the surrounding vertebral column are implicated with the occurrence of myelitis. Therefore, lesions of the surrounding vertebral column might be a predisposing factor of myelitis (Table 3 ).

Table 3. Relation between lesions of spinal cords and vertebras.
\begin{tabular}{lccc}
\hline Lesions of spinal cords & Positive & Negative & No abnormal findings \\
\hline Cervical cord & 12 & 0 & 4 \\
Thoracic cord & 5 & 8 & 11 \\
\hline
\end{tabular}

\section{Pre-existing infections in cervical and thoracic cords}

Three of the 18 patients with cervical cord lesions had pre-existing infections, while 13 patients had no such infections. Twenty patients with thoracic lesions had pre-existing infections, while nine patients had no such infections. The chi-squared test revealed a significant correlation between pre-existing infections and the occurrence of thoracic myelitis $(\mathrm{P}<0.05)$. However, no similar result was found in patients with cervical myelitis. Pre-existing infections might have more significance with respect to the onset of thoracic myelitis; however, a study using a larger sample is needed to confirm this result (Table 4).

Table 4. Pre-existing infections in cervical and thoracic cords.
\begin{tabular}{lccc}
\hline Lesions of spinal cord & Positive & Negative & Total \\
\hline Cervical & 3 & 13 & 16 \\
Thoracic & 20 & 9 & 29 \\
\hline
\end{tabular}

\section{DISCUSSION}

\section{Effects of age of onset and gender}

The incidence of AM is 1 to 4 in one million people per year, and can affect individuals of all age groups. Previous studies (TMCWG, 2002) have shown that there is no difference in gender, season, or family history in the occurrence of myelitis. However, a study conducted in California in 2012 on 150 patients with AM aged from 18 to 62 years suggested that rela- 
tively more females were affected (Borchers and Gershwin, 2012). The results obtained in the present study also indicated that more females were affected, but only if minor patients were not included. A study conducted in Canada on minors with AM indicated that the average age for $42 \%$ of affected children was 10 years, and the majority affected were male children (Banwell et al., 2009). In China, no study has yet investigated the effects of age and gender on AM in minors. In this study, 10 of the affected minor patients were male, which suggested that male minor patients might be more likely to have AM. In addition, in the group of 10 to 18 year-old, male patients affected by the disease were also in the majority. Studies using larger samples are worth conducting to further explore the phenomenon of increased AM incidence in Chinese minors.

\section{Pre-existing infections}

The mechanisms for developing AM remain unknown, and the relevant research conducted to date has been inconclusive (Misra et al., 1996; al Deeb et al., 1997; Wilmshurst et al., 1999). Approximately $40 \%$ of AM patients have a history of upper respiratory infections, diarrhea, and other viral infections or vaccinations (such as influenza or varicella-zoster). Signs and symptoms of AM are generally observed at the recovery phase of infections. No viral antibodies have been screened out from the cerebrospinal fluid of AM patients to date, and no virus has yet been extracted from these patients' nervous tissues. Therefore, AM directly caused by viral infections cannot be verified. Currently, the disease is also presumed to be caused by effects of the immune response following virus infections rather than by direct viral infections in the spinal cord. In some cases, AM was diagnosed after vaccinations that could lead to abnormal immunological responses (Sato et al., 2011). In this study, 35\% of patients had symptoms of pre-existing infections, which is lower than the $40 \%$ reported in previous studies. This might be due to neglect during the collection of the patients' medical histories or due to mild symptoms of which patients were not aware. Therefore, studies with larger samples are needed to clarify the relationship between AM and pre-existing infections.

\section{Imaging examinations}

Two interesting phenomena were found in the imaging examinations. First, $66 \%$ of patients with cervical myelitis were found to be associated with degeneration of the spinal column, most of which occurred on the same vertebral column. This was significantly higher than associated diseases of the thoracic vertebral column, suggesting that the onset of cervical myelitis was associated with degeneration of the cervical vertebrae. Immune actions are generally believed to be the main mechanism of intervertebral disk degeneration, and are also known to play an important role in the onset of myelitis. Previous studies (Wang and $\mathrm{Hu}, 1994$ ) indicated that close autoimmunity exists in type-I collagen, type-II collagen, glycoprotein, and the extracellular matrix of the lamina terminalis of cartilage in healthy tissues of inter-vertebral disks. When the inter-vertebral disk is ruptured, human lymphocytes are extremely sensitive to these components, which can lead to delayed hypersensitivity. The inter-vertebral disk is enclosed from surrounding blood circulation and is excluded from human immune actions after embryonic development with the degeneration of the inner vessels. When the inter-vertebral disk is ruptured or impaired, the nucleus pulposus is pushed through 
fibrous rings and the posterior longitudinal ligament. During the repair process, vascularization develops into the nucleus pulposus that is within the range of human immune actions. Glycoproteins and $\beta$-proteins in the matrix of the nucleus pulposus become the antigens that will induce the immunological response. Previous studies (Olmarker et al., 1993; Palmer et al., 1993; Spiliopoulou et al., 1994; Kang et al., 1997; Satoh et al., 1999; Kohyama et al., 2000; Burke et al., 2002) have shown that the degeneration of the inter-vertebral disk plays a significant role in the development of secondary impairments of spinal cords. In China, only one study has been published concerning acute cervical myelitis that supports our hypothesis that cervical inter-vertebral disk degeneration might be one of the risk factors inducing cervical myelitis (Wang et al., 2010).

The second phenomenon emerging from the imaging examination was that myelitis occurring on thoracic segments had no corresponding degeneration at the same level. Only five of the 29 patients $(17 \%)$ with thoracic myelitis were associated with degeneration of thoracic vertebrae, and 20 patients (69\%) had pre-existing infections. These results differed from those observed in patients with cervical myelitis, in which the degeneration rate was 12/18 $(66 \%)$ and there was a low rate of pre-existing infections (3/10;30\%). Therefore, we propose, for the first time, that pre-existing infections might be a risk factor or play an important role in the onset or the occurrence, respectively, of thoracic myelitis.

Finally, we attempted to interpret differences in the onset of thoracic and cervical myelitis based on the blood supply features at different sites. The blood supply to the cervical and lumbar vertebrae is abundant, whereas the blood supply to thoracic cords is limited; the area from T1 to T5 is even referred to as "the dangerous zone". According to pathological and physiological characteristics, recovery after impairment is usually more difficult at sites with less blood supply. Therefore, we suggest that immune actions might be strengthened at these sites after they suffer from infection.

\section{CONCLUSION}

$\mathrm{AM}$ is a neurological disorder with high deformity rates, and its mechanisms remain unknown. Analysis of clinical features is helpful for detecting risk factors and preventing the occurrence of AM as early as possible. Three main conclusions were reached in this descriptive study: 1) male minors might be more likely to be affected by AM; 2) degeneration of the inter-vertebral disk was commonly seen in cervical myelitis; 3 ) thoracic myelitis was relatively more associated with pre-existing infections. Further studies with increased sample sizes and control groups are necessary, along with detailed experimental studies addressing each of the conclusions above to confirm these results and to verify these relationships further.

\section{REFERENCES}

al Deeb SM, Yaqub BA, Bruyn GW and Biary NM (1997). Acute transverse myelitis. A localized form of postinfectious encephalomyelitis. Brain 120: 1115-1122.

Banwell B, Kennedy J, Sadovnick D, Arnold DL, et al. (2009). Incidence of acquired demyelination of the CNS in Canadian children. Neurology 72: 232-239.

Borchers AT and Gershwin ME (2012). Transverse myelitis. Autoimmun. Rev. 11: 231-248.

Burke JG, Watson RW, McCormack D, Dowling FE, et al. (2002). Spontaneous production of monocyte chemoattractant protein-1 and interleukin-8 by the human lumbar intervertebral disc. Spine 27: 1402-1407.

Kang JD, Stefanovic-Racic M, McIntyre LA, Georgescu HI, et al. (1997). Toward a biochemical understanding of human 
intervertebral disc degeneration and herniation. Contributions of nitric oxide, interleukins, prostaglandin E2, and matrix metalloproteinases. Spine 22: 1065-1073.

Kohyama K, Saura R, Doita M and Mizuno K (2000). Intervertebral disc cell apoptosis by nitric oxide: biological understanding of intervertebral disc degeneration. Kobe J. Med. Sci. 46: 283-295.

Matsumoto M, Fujimura Y, Suzuki N, Nishi Y, et al. (1998). MRI of cervical intervertebral discs in asymptomatic subjects. J. Bone Joint Surg. Br. 80: 19-24.

Misra UK, Kalita J and Kumar S (1996). A clinical, MRI and neurophysiological study of acute transverse myelitis. $J$. Neurol. Sci. 138: 150-156.

Olmarker K, Rydevik B and Nordborg C (1993). Autologous nucleus pulposus induces neurophysiologic and histologic changes in porcine cauda equina nerve roots. Spine 18: 1425-1432.

Palmer RM, Hickery MS, Charles IG, Moncada S, et al. (1993). Induction of nitric oxide synthase in human chondrocytes. Biochem. Biophys. Commun. 193: 398-405.

Sá MJ (2009). Acute transverse myelitis: a practical reappraisal. Autoimmun. Rev. 9: 128-131.

Sato N, Watanabe K, Ohta K and Tanaka H (2011). Acute transverse myelitis and acute motor axonal neuropathy developed after vaccinations against seasonal and 2009 A/H1N1 influenza. Intern. Med. 50: 503-507.

Satoh K, Konno S, Nishiyama K, Olmarker K, et al. (1999). Presence and distribution of antigen-antibody complexes in the herniated nucleus pulposus. Spine 24: 1980-1984.

Spiliopoulou I, Korovessis P, Konstantinou D and Dimitracopoulos G (1994). IgG and IgM concentration in the prolapsed human intervertebral disk sand sciatica etiology. Spine 19: 1320-1323.

Transverse Myelitis Consortium Working Group (2002). Proposed diagnostic criteria and nosology of acute transverse myelitis. Neurology 59: 499-505.

Wang KG and Hu YG (1994). Lumbar disk herniation autoimmune research. J. Bone Joint Surgery 14: 259-262.

Wang LQ, Zhang XQ and Zhou H (2010). The study of relationship between acute cervical myelitis and cervical disk degeneration. Chin. J. Neuro Immunol. Neurol. 17: 358-360.

Wilmshurst JM, Walker MC and Pohl KR (1999). Rapid onset transverse myelitis in adolescence: implications for pathogenesis and prognosis. Arch. Dis. Child. 80: 137-142.

Zettl UK, Stuve O and Patejdl R (2012). Immune-mediated CNS diseases: a review on nosological classification and clinical features. Autoimmun. Rev. 11: 167-173.

Zhang CY (2010). Anthropotomy (M). People's Medical Publishing House, 1716-1717. 